\title{
Repeated intra-nigrostriatal injection of phorbol myristate acetate induces microglial senescence in adult rats
}

\author{
LIN LIU $^{1}$, XIAO-GUANG LUO ${ }^{1,2}$, HONG-MEI YU $^{1}$, YU FENG $^{1}$, \\ YAN REN $^{1}$, YA-FU YIN ${ }^{3}, \mathrm{HONG} \mathrm{SHANG}^{2}$ and ZHI-YI HE ${ }^{1}$ \\ Departments of ${ }^{1}$ Neurology, ${ }^{2}$ Laboratory Medicine and ${ }^{3}$ Nuclear Medicine, \\ The First Affiliated Hospital of China Medical University, Shenyang, Liaoning 110001, P.R. China
}

Received November 21, 2014; Accepted August 17, 2015

DOI: $10.3892 / \mathrm{mmr} .2015 .4412$

\begin{abstract}
Phorbol myristate acetate (PMA), as a potent tumor promoter, may induce microglial senescence. The present study investigated the effect of PMA infection on microglial senescence. From 58 male Sprague-Dawley rats, 10 were randomly selected and divided into a PMA injection group, containing five rats $(0.5 \mu \mathrm{g} / \mu \mathrm{l}$ PMA) and a control group, containing five rats (commensurable $0.9 \%$ saline). Immunofluorescent staining of Iba-1 and enzyme-linked immunosorbent assay analyses of the expression levels of tumor necrosis factor (TNF)- $\alpha$ and interleukin (IL) $-1 \beta$ were performed in these two groups. The remaining 48 rats were randomly divided into the following three groups, each containing 16 rats: Repeated injection control group (commensurable normal saline, once a week for 4 weeks), single PMA injection group $(0.5 \mu \mathrm{g} / \mu 1 \mathrm{PMA}$, once in the first week) and repeated injection PMA group (0.5 $\mu \mathrm{g} / \mu 1$ PMA, once a week for 4 weeks). The expression levels of p21, detected using double immunofluorescence staining with Iba-1, and $\beta$-galactosidase, via double immunohistochemical staining of Iba-1, were examined in these three groups. The results indicated that a single injection of PMA did not change the microglial morphology and had no significant effects on the expression levels of TNF- $\alpha$ and IL-1 $\beta$, compared with the control group $(\mathrm{P}>0.05)$. Following four repeated injections of PMA, the microglia in the substantia nigra presented with features of senescence, characterized by increased expression levels of $\beta$-galactosidase $(\mathrm{P}<0.001)$ and $\mathrm{p} 21(\mathrm{P}<0.001)$, compared with the repeated injection control group. In conclusion, repeated intra-nigrostriatal treatment with PMA induced microglial senescence with increased
\end{abstract}

Correspondence to: Dr Xiao-Guang Luo, Department of Neurology, The First Affiliated Hospital of China Medical University, 155 Nanjing Bei Street, Heping, Shenyang, Liaoning 110001, P.R. China

E-mail: xiaoguangluoo@163.com

Key words: microglia, senescence, substantia nigra, phorbol myristate acetate expression levels of $\beta$-galactosidase and p21 in the substantia nigra of the rats.

\section{Introduction}

Microglia are one of the resident populations of mononuclear phagocytes in the central nervous system (CNS) and account for $10 \%$ of the total glial cell population in the brain (1). Increasing evidence has suggested that microglia have neurotoxic effects when they are overactivated following severe injury or during neurodegenerative disease $(2,3)$. The neurotoxic effects of the activated microglia in the aged neurodegenerative brain may result in age-associated microglia senescence (4). Microglia senescence, which underlies the alterations of microglial function and incorrect responses to stimuli, can promote eventual neurodegeneration $(5,6)$. Emerging evidence has indicated that microglial senescence can contribute to age-associated neurodegenerative disease, including Parkinson's disease (PD), which is characterized by the degeneration of dopaminergic neurons in the substantia nigra pars compacta (7-9). The most prominent features of microglial senescence are morphological alterations, described as dystrophy, and alterations in the inflammatory profile $(4,7)$. In addition, a previous study indicated that the expression levels of proinflammatory cytokines, including tumor necrosis factor (TNF)- $\alpha$ and interleukin (IL) $-1 \beta$ are increased in the brains of senescence-accelerated mice (10).

Senescence is a state of irreversible cell withdrawal from the proliferative pool, and occurs at the exhaustion of the proliferative lifespan (11). Cellular senescence is characterized by elevated endogenous $\beta$-galactosidase activity, which is commonly used as a marker for cellular senescence (12). In addition, the induction of $\mathrm{p} 21$, the first identified inhibitor of cyclin/cyclin-dependent kinase complexes, is essential for the onset of cell cycle arrest in cell senescence $(13,14)$. Senescence can also be caused by pathological stimulation, including the activation of oncogenes (oncogene-induced senescence; OIS) (15). Phorbol myristate acetate (PMA), commonly known as 12-O-Tetradecanoylphorbol-13-acetate, is a potent tumor promoter, which is often applied in biomedical investigations to activate the signal transduction enzyme, protein kinase $\mathrm{C}$ (PKC) $(16,17)$, which is involved in oncogene activation. In addition, it has also been reported that senescence can be 
driven by the activation of PKC (18-20). Therefore, the present study hypothesized that PMA, as a carcinogen, can also induce microglial senescence. In the present study, the expression levels of TNF- $\alpha$, IL-1 $\beta, \beta$-galactosidase and p 21 in the substantia nigra of rats were investigated, as were the effects on these levels of expression following PMA administration

\section{Materials and methods}

Animals. Male Sprague-Dawley rats (12 weeks old; 220-260 g) were provided by the Biological Science Animal House (Liaoning, China). The animals were maintained in a temperature-controlled environment at $22-24^{\circ} \mathrm{C}$ on a $12: 12 \mathrm{~h}$ light-dark cycle, and were provided with free access to food and water. All animal experiments were performed in accordance with the National Institutes of Health Guide for the Care and Use of Laboratory Animals (National Institutes of Health, Bethesda, MA, USA) and the present study was approved [no. SCXK (Liao) 2008-0005] by the ethics committee of China Medical University (Shenyang, China).

Treatment groups. A total of 58 rats were used in the present study. Firstly, 10 of the 58 rats were randomly selected and divided into two groups: PMA injection group $(n=5)$ and control group $(n=5)$. In each group, injection was performed once, and all 10 rats were sacrificed by an intracardiac injection of sodium pentobarbital ( $75 \mathrm{mg}$ of $390 \mathrm{mg} / \mathrm{ml}$ solution) 1 week following injection to determine whether a single PMA injection allowed the microglia to enter a resting state. In the control group, normal saline (NS; 0.9\%saline solution), rather than PMA, was injected providing a single injection control group. The remaining 48 rats were randomly divided into three groups: i) repeated injection control group $(n=16)$; ii) single PMA injection group $(n=16)$ and iii) repeated PMA injection group $(n=16)$. In the single PMA injection group, the rats were injected with PMA once in the first week. For the repeated PMA injection group, the rats were injected with PMA four times, once a week. In the repeated injection control group, NS, rather than PMA, was injected four times, once a week. All 48 rats were sacrificed, as described above, at the fifth week following the first injection (Fig. 1).

Intra-nigrostriatal injection of PMA. To observe the microglial changes in the substantia nigra, PMA (Sigma-Aldrich, St. Louis, MO, USA) was injected into the substantia nigra by performing stereotaxic surgery. For the stereotaxic surgery, the rats were anesthetized with an intraperitoneal injection of pentobarbital $(50 \mathrm{mg} / \mathrm{kg}$; Apoteksbolaget, Stockholm, Sweden). Once the animals were deeply anesthetized, they were placed in a stereotaxic apparatus (David Kopf Instruments, Tujunga, CA, USA). Subsequently, the rats were injected with a sub-toxic concentration of PMA $(0.5 \mu \mathrm{g} / \mu \mathrm{l}$; $2 \mu \mathrm{l}$ at each site, into the right nigrostriatal pathway (medial forebrain bundle) at stereotaxic coordinates (anteroposteriorly $4.4 \mathrm{~mm}$ from the bregma; mediolaterally $+1.0 \mathrm{~mm}$ from the midline; dorsoventrally $-7.2 \mathrm{~mm}$ from the skull), as adapted from Grealish et al (21). The control group was injected with the same volume of NS ( $2 \mu 1$ at each site). At the end of each injection, the syringe needle remained in position for $5 \mathrm{~min}$ and was then slowly withdrawn to prevent solution reflux.
Tissue sample collection. Following the appropriate time-periods post-injection, the animals were deeply anesthetized with pentobarbital. A number of the brains from the PMA injection group $(n=3)$ and control group $(n=3)$ were immediately removed, and the whole striatum and substantia nigra were rapidly dissected and placed on the ice, which was used for enzyme-linked immunosorbent assay (ELISA) analysis of TNF- $\alpha$ and IL-1 $\beta$. The remaining animals were perfused through the aorta with $0.9 \%$ saline, followed by ice-cold fixative consisting of $4 \%$ paraformaldehyde (Sigma-Aldrich,) in $100 \mathrm{mM}$ phosphate-buffered saline (PBS). The brains were then dissected (3-4 mm thickness) and post-fixed for $24 \mathrm{~h}$ with the same fixative. Following fixation, a number of the brain samples were transferred into $15 \%$ sucrose (Sigma-Aldrich) solution overnight at $4^{\circ} \mathrm{C}$, and were subsequently to a $30 \%$ sucrose solution until the brain samples sunk to the tube bottom. Subsequently, brain samples from all the remaining animals in the PMA injection group and control group, and samples from the animals in the other three groups were sectioned using a cryostat (CM3050S; Leica Microsystems GmbH, Nussloch, Germany) at a thickness of $25 \mu \mathrm{m}$. These sections were then mounted onto poly-l-lysine-coated slides (CEL Associates, Pearland, TX, USA), which were prepared for immunofluorescence. The remaining brain samples ( $n=8 /$ group) from the repeated injection control group, single PMA injection group and repeated PMA injection group were embedded in paraffin (Sigma-Aldrich), and 5- $\mu \mathrm{m}$ coronal sections were obtained using a microtome, which were processed for $\beta$-galactosidase staining and immunohistochemistry.

Assessment of the effect of single PMA injection on microglia. To determine the effect of a single PMA injection on the microglia, immunofluorescence staining for Iba-1 was performed, and the levels of TNF- $\alpha$ and IL- $1 \beta$ were detected using ELISA. The brain samples from the rats in the PMA injection group and control group were used for this assessment. For the immunofluorescence staining, the frozen sections from the PMA injection group and control group were permeabilized using $0.3 \%$ Triton X-100/PBS (Sigma-Aldrich) for $10 \mathrm{~min}$ at room temperature. Following hydration with ethanol and being fixed with $10 \%$ formaldehyde, the sections were microwave-heated for $6 \mathrm{~min}$ in sodium citrate buffer (10 mM; pH 6.0) for antigen retrieval. The sections were then blocked with $10 \%$ bovine serum albumin (Sigma-Aldrich) in PBS for $30 \mathrm{~min}$. The sections were incubated overnight with polyclonal rabbit anti-rat Iba-1 (cat. no. 019-19741; 1:100; Wako Pure Chemicals Industries, Osaka, Japan) at $4^{\circ} \mathrm{C}$. Following several washes with PBS, the sections were incubated for $2 \mathrm{~h}$ at room temperature with Alexa-488 (green fluorescence)-conjugated goat anti-rabbit IgG polyclonal antibody (cat. no. A-11034; 1:800; Invitrogen Life Technologies, Carlsbad, CA, USA). Fluorescence images were captured using a digital camera attached to a fluorescent inverted microscope (Nikon Eclipse E1000; Nikon Corporation, Tokyo, Japan). Green color indicated positive expression of Iba-1.

The target proteins of TNF- $\alpha$ and IL- $1 \beta$ were extracted, according to the manufacturer's instruction of the Rat TNF- $\alpha$ and IL-1 $\beta$ ELISA kits (Invitrogen Life Technologies). Briefly, the samples were homogenized in lysis buffer containing 


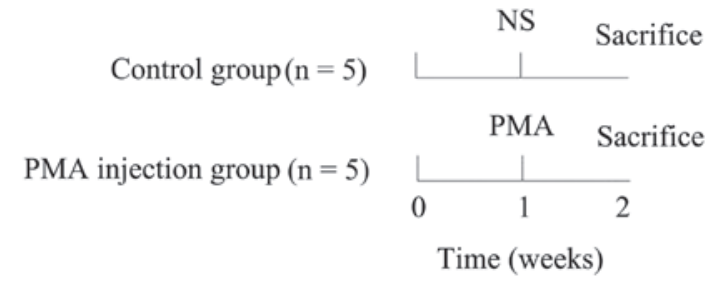

\begin{tabular}{|c|c|c|}
\hline Single time PMA injection group $L$ & $\begin{array}{c}\text { PMA } \\
\mid\end{array}$ & Sacrifice \\
\hline$(\mathrm{n}=16) 0$ & 1 & 5 \\
\hline
\end{tabular}

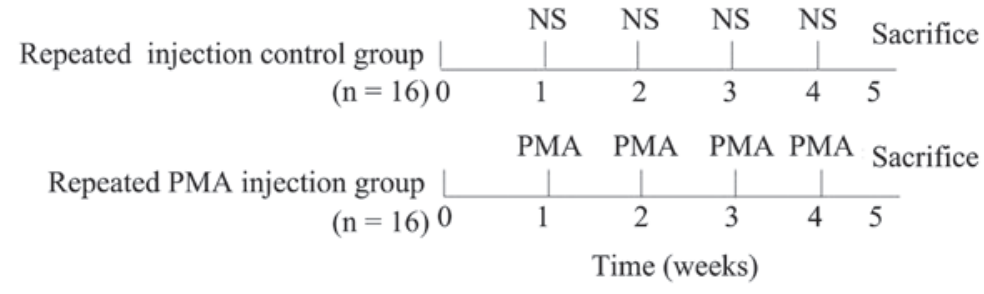

Figure 1. Administration procedure in each group. In the upper two procedures, the animals received a single injection of PMA in the PMA injection group and a commensurable NS in control group, and were sacrificed 1 week post-injection. In the lower three procedures, the animals received a single injection of PMA in the first week in the single time PMA injection group. PMA injections or commensurable NS were performed once a week four times in the remaining two groups. The animals in these three groups were sacrificed at the fifth week. PMA, phorbol myristate acetate; NS, normal saline.

protease and phosphatase inhibitors, and $1 \%$ Triton $\mathrm{X}-100$, and then centrifuged at $13,000 \mathrm{x} \mathrm{g}$ for $30 \mathrm{~min}$ at $4^{\circ} \mathrm{C}$. The resultant supernatants were collected and frozen at $-80^{\circ} \mathrm{C}$ until analysis. For each reaction in the 96 -well plate, $50 \mu \mathrm{g}$ of proteins were used, and ELISA were performed according to the manufacturer's instructions, as described by Koziorowski et al (22). The total proteins were extracted using RIPA lysis buffer (Beyotime Institute of Biotechnology, Haimen, China). Following centrifugation, the supernatant was loaded onto precoated DNA-binding protein wells for ELISA. The absorbance was measured at $450 \mathrm{~nm}$ on an MRX 96-well plate reader (Dynex Technologies, Inc., Chantilly, VA, USA).

Assessment of the effect of repeated PMA injection on microglia. The expression of p21 was measured using double immunofluorescence staining with Iba-1. For double immunofluorescence, following the permeabilization, hydration, fixation, antigen retrieval and blocking steps described above, sections from the repeated injection control group, single PMA injection group and repeated PMA injection group were incubated with a mixture of polyclonal rabbit anti-rat Iba-1 (cat. no. 019-19741; 1:1,00; Wako Pure Chemicals Industries) and polyclonal mouse anti-rat p21 (cat. no. ab80633; 1:80; Abcam, Cambridge, UK) primary antibodies followed by a mixture of Alexa-488 (green fluorescence) and Alexa-594 (red fluorescence)-conjugated polyclonal goat anti-rabbit IgG (cat. no. A-11034; 1:800) and polyclonal goat anti-mouse IgG (cat. no. A-11029; 1:1,000; Invitrogen Life Technologies). The red staining of the positive expression of $\mathrm{p} 21$ enabled differentiation from the green positive expression of Iba-1.

Double staining for $\beta$-galactosidase and Iba- 1 was performed, as previously described by Itahana et al (23). Following dewaxing and hydration, the rest sections from the repeated injection control group, single PMA injection group and repeated PMA injection group were also permeabilized for $10 \mathrm{~min}$ and blocked for another $30 \mathrm{~min}$, as described above. The sections were then incubated overnight at $37^{\circ} \mathrm{C}$ without $\mathrm{CO}_{2}$ in freshly prepared staining buffer, containing $1 \mathrm{mg} / \mathrm{ml} \mathrm{X-gal,} 40 \mathrm{mM}$ citric acid/sodium phosphate (pH 6.0), $5 \mathrm{mM}$ potassium ferricyanide, $150 \mathrm{mM} \mathrm{NaCl}$ and $2 \mathrm{mM} \mathrm{MgCl}_{2}$ (all purchased from Sigma-Aldrich), which was substituted with normal saline prior to observation. Following $\beta$-galactosidase staining, immunohistochemical staining of Iba-1 was performed according to the avidin-biotin-peroxidase complex method (Vector Laboratories, Burlingame, CA, USA). Briefly, the stained sections were incubated overnight at $4^{\circ} \mathrm{C}$ with polyclonal rabbit anti-rat Iba-1 antibody (cat. no. 019-19741; 1:100; Wako Pure Chemicals Industries) and then with biotinylated polyclonal goat antirabbit IgG antibody (cat. no. A-11034; 1:800; Invitrogen Life Technologies) for $30 \mathrm{~min}$ at room temperature, followed by horseradish peroxidase-conjugated avidin for $30 \mathrm{~min}$ at room temperature. Finally, the colors were developed with 3-amino-9-ethylearbazole substrate (Vector Laboratories, Inc.) for Iba-1. The positive $\beta$-galactosidase cells were stained blue, enabling differentiation from the red-brown color of the Iba-1-positive cells.

Unbiased stereological estimation of the number of cells were performed in all double staining sections throughout the substantia nigra area using StereoInvestigator analysis software (version 6.0; MicroBrightField, Williston, VT, USA) combined with a Nikon Eclipse E600 microscope (Nikon Corporation) and the optical fractionator method, according to previously published reports $(24,25)$. The boundaries of the substantia nigra were defined according to previously published anatomical analysis in rats (area of counting frame, 64,000 $\mathrm{mm}^{3}$; guard height, $2 \mu \mathrm{m}$; spaced $300 \mu \mathrm{mm}$ apart in the $\mathrm{x}$-direction, and $200 \mu \mathrm{m}$ apart in the $\mathrm{y}$-direction) (26), and the cells were counted in 24 sections ( $n=8$ for each group) along the entire substantia nigra, including pars reticulate and compacta. The cells were counted by an observer in a blinded-manner.

For identifying the double stained-positive senescent microglias, 10 high power microscopic fields throughout 

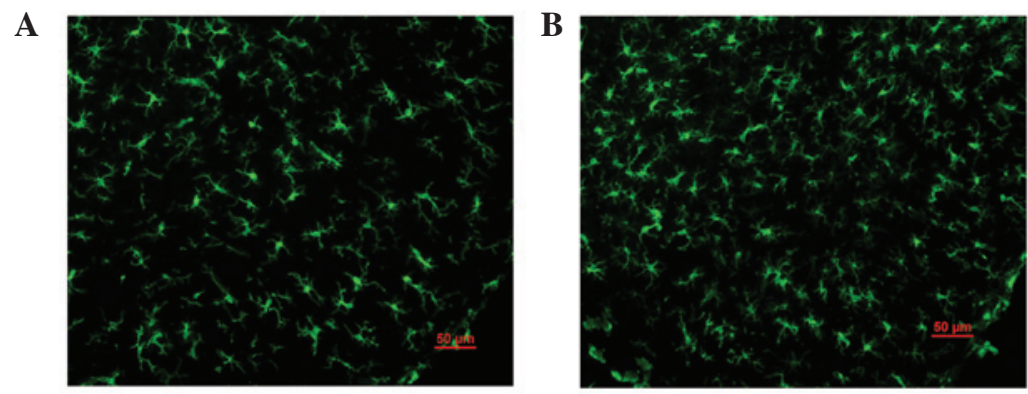

C

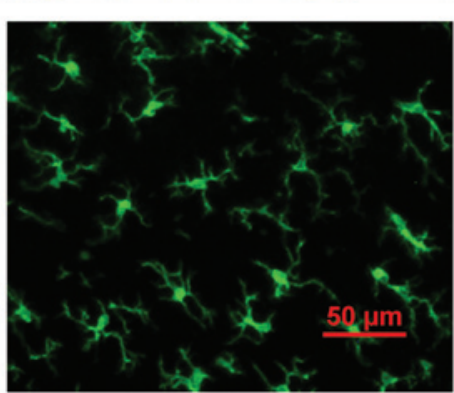

Control group

D

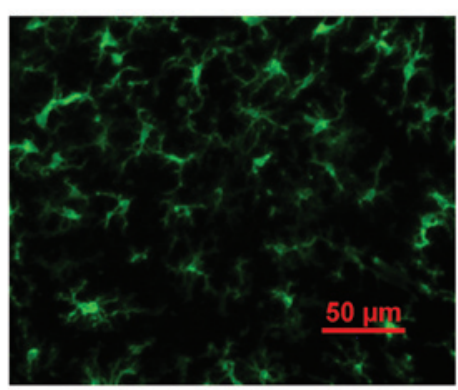

PMA injection group

Figure 2. Immunofluorescence staining of Iba-1 in the microglia in the substantia nigra 1 week following a single PMA injection. Immunofluorescence of Iba-1 revealed that, compared with (A) control group, microglia in the (B) PMA injection group were in a resting state, with typical ramified branches. (C and D) Enlarged images of A and B, respectively. PMA, phorbol myristate acetate.

the substantia nigra were randomly selected. The color of the double-positive cells of Iba- 1 and $\beta$-galactosidase was confirmed as violet-black, which formed from the merge of blue ( $\beta$-galactosidase) and red-brown (Iba-1). The color of the double-positive cells of Iba-1 and p21 was defined as yellow, which formed from the merge of red (p21) and green (Iba-1). Only the microglias with clearly visible cell bodies were counted. The percentage of senescent microglia in each high power field (magnification, x400) was presented as a graph.

Statistical analysis. Data are presented as the mean \pm standard error of the mean. Differences between groups were determined using one-way analysis of variance followed by Bonferroni's t-test for multiple comparisons. All data were analyzed using SPSS 11.5 software (SPSS, Inc., Chicago, IL, USA). $\mathrm{P}<0.05$ were considered to indicate a statistically significant difference.

\section{Results}

Resting state of microglia 1 week following single PMA injection. As shown in Fig. 2, the majority of the microglia were in the resting state with typical ramified branches in the PMA-injected group (Figs. 2B and D). Analysis of ELISA demonstrated no significant difference in the expression levels of TNF- $\alpha$ or IL-1 $\beta$ between the control group and PMA injected group $(\mathrm{P}>0.05$; Fig. 3$)$. These results indicated that PMA had no significant effect on the microglia following a single injection at one week.

Expression of $\beta$-galactosidase is increased in microglia following repeated injections of PMA. Images of the double immunofluorescence staining are shown in Fig. 4. There were more double-stained positive cells in the repeated PMA injection group, compared with the repeated injection control

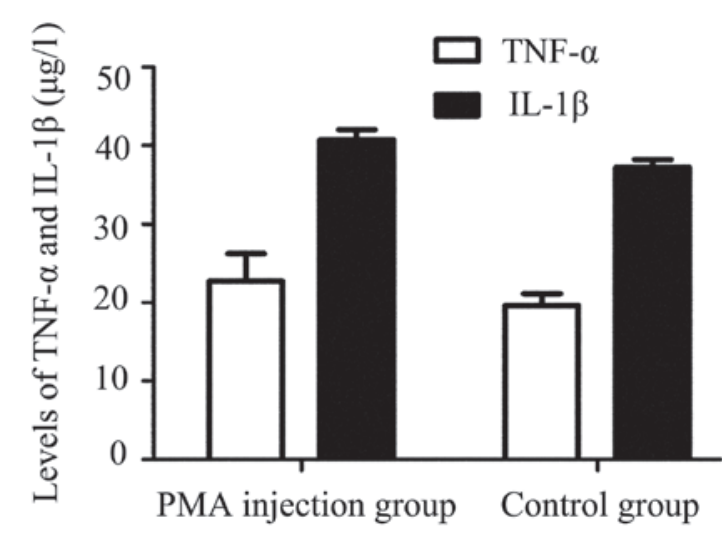

Figure 3. Expression of TNF- $\alpha$ and IL-1 $\beta$ between the PMA injection group and the control group. No significant differences were observed in the expression levels of TNF- $\alpha$ or IL-1 $\beta$ between the PMA injection group and the control group $(\mathrm{P}=0.469)$. Data are presented as the mean \pm standard error of the mean. PMA, phorbol myristate acetate; TNF, tumor necrosis factor; IL, interleukin.

group $(\mathrm{P}<0.001)$ and single $\mathrm{PMA}$ injection group $(\mathrm{P}=0.002)$, as shown in Fig. 5. However, the numbers of double-stained positive cells in the single PMA injection group were not significantly increased, compared with those in the repeated injection control group $(\mathrm{P}=0.777)$. The percentage of doublestained positive cells in the repeated PMA injection group $(17.15 \pm 4.25 \%)$ was significantly increased, compared with the repeated injection control group $(10.34 \pm 1.86 \% ; \mathrm{P}<0.001)$ and single PMA injection group $(11.32 \pm 1.76 \%$; $\mathrm{P}=0.002)$, respectively. These results suggested that repeated injection of PMA increased the expression of $\beta$-galactosidase in the microglia in the substantia nigra of the rats.

Expression of p21 increases in microglia following repeated injections of PMA. Representative double-staining images 
A

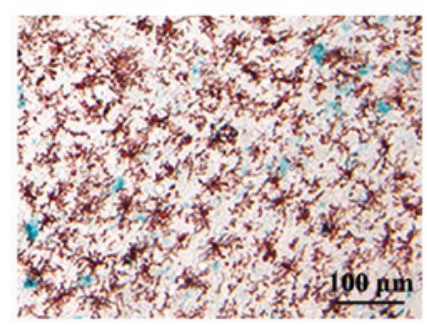

D

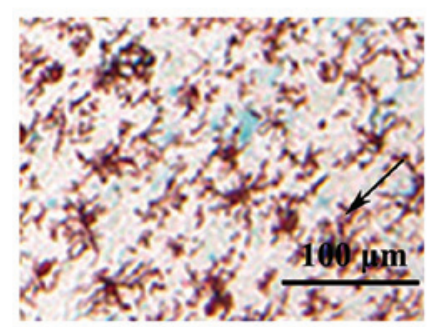

Repeated injection control group
B

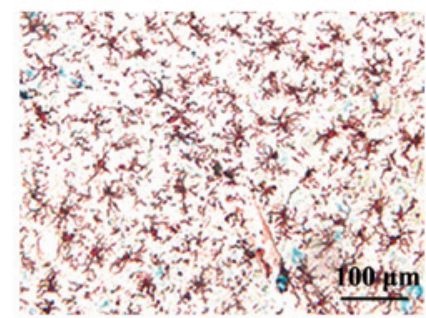

$\mathbf{E}$

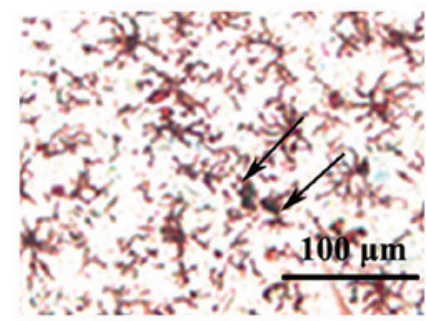

Single PMA injection group
C

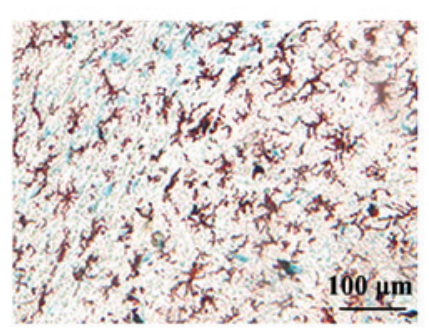

F

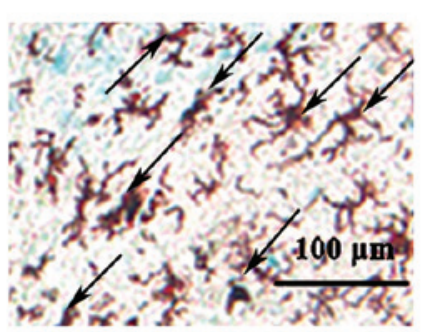

Repeated PMA injection group

Figure 4. Double staining for Iba-1 and $\beta$-galactosidase in the substantia nigra. Double-staining revealed that $\beta$-galactosidase was colocalized with Iba-1 in each group. Compared with the (A) repeated injection control group or (B) single PMA injection group, more double-staining positive cells were present in the (C) repeated PMA injection group. (D-F) Enlarged images of A-C, respectively. The black arrows indicated double-stained positive cells (violet-black). The blue color represents the positive expression of $\beta$-galactosidase. The red-brown represents the positive expression of Iba-1. PMA, phorbol myristate acetate.

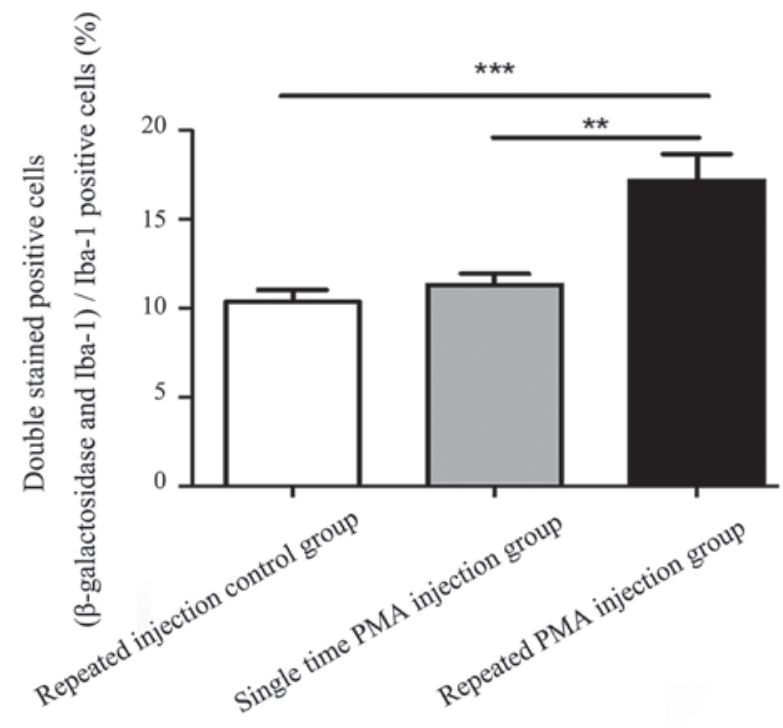

Figure 5. Percentages of $\beta$-galactosidase and Iba-1-positive cells, compared with Iba-1-positive cells. A higher percentage of double-staining positive cells were observed in the repeated PMA injection group, compared with the repeated injection control group or the single PMA injection group. ${ }^{* *} \mathrm{P}<0.01$ and ${ }^{* * *} \mathrm{P}<0.001$. Data are presented as the mean \pm standard error of the mean. PMA, phorbol myristate acetate.

of p21 and Iba-1 are shown in Fig. 6A-C. The numbers of microglia exhibiting positive expression of p21 were significantly higher in the repeated PMA injection group, compared with the repeated injection control group $(\mathrm{P}<0.001)$ and the single PMA injection group $(\mathrm{P}<0.001)$, as shown in Fig. 6D. The numbers of double-stained positive cells in the single PMA injection group were not significantly increased, compared to the repeated injection control group $(\mathrm{P}=0.912)$. The percentage of double-stained positive cells in the repeated PMA injection group $(16.67 \pm 2.17 \%)$ was significantly increased, compared with the repeated injection control group $(11.31 \pm 1.76 \%$; $\mathrm{P}<0.001)$ and single PMA injection group $(11.64 \pm 1.87 \%$; $\mathrm{P}<0.001)$, respectively. These results indicated that repeated injection of PMA increased the expression of p21 in the microglia in the substantia nigra of the rats.

\section{Discussion}

In the present study, the effect of intra-nigrostriatal injection of PMA on microglia senescence was investigated. The results demonstrated that PMA had no significant effect on the microglia following a single injection at one week. Following four repeated injections of PMA, microglia in the substantia nigra exhibited certain senescence characteristics, including increased expression levels of $\beta$-galactosidase and $\mathrm{p} 21$. These results demonstrated that repeated injection of PMA resulted in microglia senescence, whereas a single injection of PMA did not induce microglia senescence in the substantia nigra of the rats.

Microglia senescence is manifested by morphological changes and alterations in inflammatory profile (4). The observation of morphological alterations, characterized by cytoplasmic fragmentation, twisting processes and clusters, has been used in several studies to identify dystrophic microglia $(27,28)$. In the present study, the majority of the microglia presented with no dystrophic microglia features in the PMA injection group, indicating that a single PMA injection did not lead to morphological changes, which is one of the characteristics of microglia senescence. Microglia belong to the macrophage lineage and are the predominant form of active immune defense in the CNS. TNF- $\alpha$ and IL-1 $\beta$ are two predominant proinflammatory cytokines produced by microglia during CNS inflammation (29). In rodents, reports have suggested that production of the TNF- $\alpha$ and IL-1 $\beta$ proinflammatory cytokines are increased in the brain of the 
A

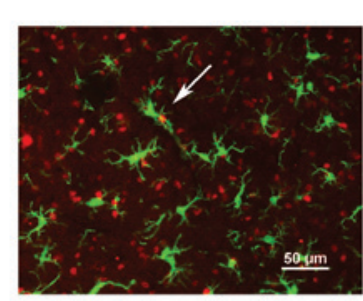

B

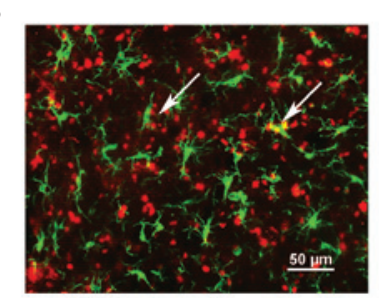

C

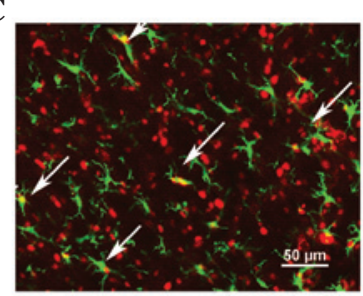

D

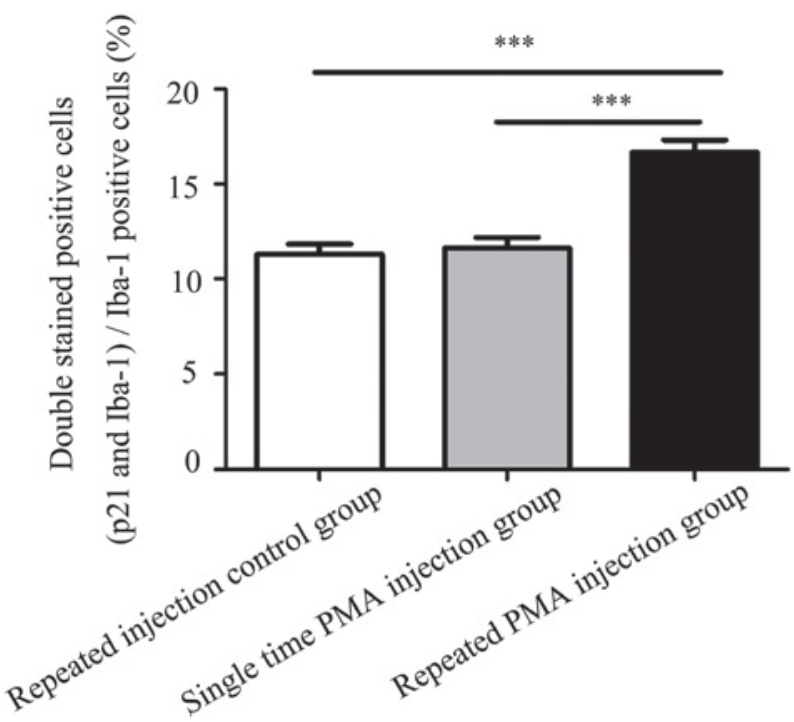

Figure 6. Immunofluorescence staining for Iba-1 and p21 in the substantia nigra. Compared with the (A) repeated injection control group or (B) single PMA injection group, a higher percentage of cells positive for p21 and Iba-1 were identified in the (C) repeated PMA injection group. (D) Percentages of p21 and Iba-positive cells compared with Iba-1 positive cells in the different groups. The white arrows indicate double-stained positive cells (yellow). The red color represents positive expression of p21 and the green colour represents positive expression of Iba-1A. A higher percentage of double-staining positive cells were observed in the repeated PMA injection group, compared with the repeated injection control group or the single PMA injection group $\left.{ }^{(* * *} \mathrm{P}<0.001\right)$. Data are presented as the mean \pm standard error of the mean. PMA, phorbol myristate acetate.

senescence accelerated mouse $(10,30)$, while others have suggested that the proinflammatory cytokines are decreased in macrophages with aging (31-33). The results of the present study demonstrated that the levels of TNF- $\alpha$ and IL-1 $\beta$ were not significantly altered 1 week following a single PMA injection. These results suggested that microglia senescence did not occur in the substantia nigra 1 week-post single PMA injection.

Previous studies have reported that the expression level of $\beta$-galactosidase is increased during senescence $(12,34)$. In addition, the expression of $\mathrm{p} 21$ is increased during cell senescence $(13,14)$. A previous study found that, in response to repeated lipopolysaccharide administration to mimic chronic inflammation, cultured BV2 microglial cells exhibited signs of senescence, including growth arrest and enhanced $\beta$-galactosidase activity (35). In the present study, repeated administration of PMA increased the expression levels of $\beta$-galactosidase and $\mathrm{p} 21$ in the substantia nigra of the rats, which indicated that repeated administration of PMA induced microglia senescence in the substantia nigra of the rats. PMA, a potent tumor promoter, can activate $\mathrm{PKC}$ in vivo and in vitro by binding to $\mathrm{PKC}$, resulting in several types of cellular effects $(16,36)$. Studies have indicated that PMA can activate oncogenes, including Ras, in vivo and in vitro $(37,38)$. In addition, Serrano et al first reported that the expression of oncogenic Ras results in a permanent G1 arrest in primary human or rodent cells in OIS (39). Studies have also indicated that the accumulation of p21 can mediate G1 arrest (40).
Several studies also have demonstrated that the activation of oncogenes can induce OIS, and melanocytic nevi provide a clear example of OIS $(15,41-43)$. Thus, PMA induced microglia senescence may be associated with oncogenic Ras-induced G1 arrest, which also mediated by $\mathrm{p} 21$ in OIS.

PMA can also lead to oxidative stress in vitro (44). Studies have demonstrated that various forms of oxidative stress can induce senescence, including exposure to reactive oxygen species (45-47). Cultured astrocytes can undergo cellular senescence with the development of characteristics of senescence, including growth arrest, expression of $\beta$-galactosidase and increased expression of the cell-cycle inhibitor p21 in response to a variety of stressors, including oxidative stress (48-50). Thus, oxidative stress-induced senescence may be another potential mechanism of PMA-induced microglia senescence.

PMA, as a carcinogen, can induce activation of endogenic oncogenes, and resulted in microglia senescence in the substantia nigra in the present study. Oncogenes can be activated by various stimulants, which can cause tumorigenesis (51). Microglia senescence is a major factor contributing to the development of age-associated neurodegenerative diseases (52). There is a close connection between tumorigenesis and neurodegeneration (53), PD, as a neurodegenerative disease, has been reported to be associated with a decreased risk of developing cancer $(54,55)$. Therefore, the result of the present study that PMA induced microglia senescence, 
provided further evidence supporting the interaction between tumorigenesis and neurodegeneration.

In conclusion, the present study demonstrated that repeated intra-nigrostriatal treatment with PMA for carcinogen stimulation induced microglia senescence, which may be associated with OIS- and oxidative stress-induced senescence. In addition, these results provide novel evidence for the link between tumorigenesis and neurodegeneration.

\section{Acknowledgements}

This study was funded by the China National Nature Science Fund (grant. nos. 30973153, 81371421 and 81000623), the Foundation of the Liaoning Educational Committee (grant. nos. L202013136 and L2010560), the Liaoning Doctoral Starting Fund (grant. no. 20071042) and the Liaoning S\&T Project Fund (grant. no. 2011225020).

\section{References}

1. Kielian T: Microglia and chemokines in infectious diseases of the nervous system: Views and reviews. Front Biosci 9: 732-750, 2004

2. Kanaan NM, Kordower JH and Collier TJ: Age and region-specific responses of microglia, but not astrocytes, suggest a role in selective vulnerability of dopamine neurons after 1-methyl-4-phenyl-1,2,3,6-tetrahydropyridine exposure in monkeys. Glia 56: 1199-1214, 2008

3. Overmyer M, Helisalmi S, Soininen H, Laakso M, Riekkinen P Sr and Alafuzoff I: Reactive microglia in aging and dementia: An immunohistochemical study of postmortem human brain tissue. Acta Neuropathol 97: 383-392, 1999.

4. Luo XG, Ding JQ and Chen SD: Microglia in the aging brain: Relevance to neurodegeneration. Mol Neurodegener 5: 12, 2010.

5. Sawada M, Sawada H and Nagatsu T: Effects of aging on neuroprotective and neurotoxic properties of microglia in neurodegenerative diseases. Neurodegener Dis 5: 254-256, 2008.

6. Conde JR and Streit WJ: Effect of aging on the microglial response to peripheral nerve injury. Neurobiol Aging 27: 1451-1461, 2006

7. Streit WJ, Sammons NW, Kuhns AJ and Sparks DL: Dystrophic microglia in the aging human brain. Glia 45: 208-212, 2004.

8. Streit WJ, Miller KR, Lopes KO and Njie E: Microglial degeneration in the aging brain-bad news for neurons? Front Biosci 13: 3423-3438, 2008.

9. Olanow CW and Tatton WG: Etiology and pathogenesis of Parkinson's disease. Annu Rev Neurosci 22: 123-144, 1999.

10. Tha KK, Okuma Y, Miyazaki H, Murayama T, Uehara T, Hatakeyama R, Hayashi Y and Nomura Y: Changes in expressions of proinflammatory cytokines IL-1beta, TNF-alpha and IL-6 in the brain of senescence accelerated mouse (SAM) P8. Brain Res 885: 25-31, 2000.

11. Campisi J: The biology of replicative senescence. Eur J Cancer 33: 703-709, 1997.

12. Lee BY, Han JA, Im JS, Morrone A, Johung K, Goodwin EC, Kleijer WJ, DiMaio D and Hwang ES: Senescence-associated beta-galactosidase is lysosomal beta-galactosidase. Aging Cell 5: 187-195, 2006

13. Roninson IB: Oncogenic functions of tumour suppressor p21 (Waf1/Cip1/Sdi1): Association with cell senescence and tumour-promoting activities of stromal fibroblasts. Cancer Lett 179: 1-14, 2002

14. Macip S, Igarashi M, Fang L, Chen A, Pan ZQ, Lee SW and Aaronson SA: Inhibition of p21-mediated ROS accumulation can rescue p21-induced senescence. EMBO J 21: 2180-2188, 2002.

15. Campisi J and d'Adda di Fagagna F: Cellular senescence: When bad things happen to good cells. Nat Rev Mol Cell Biol 8: 729-740, 2007.

16. Blumberg PM: Protein kinase $\mathrm{C}$ as the receptor for the phorbol ester tumor promoters: Sixth Rhoads memorial award lecture. Cancer Res 48: 1-8, 1988.

17. Li YH, Bi HC, Huang L, Jin J, Zhong GP, Zhou XN and Huang M: Phorbol 12-myristate 13-acetate inhibits P-glycoprotein-mediated efflux of digoxin in MDCKII-MDR1 and Caco-2 cell monolayer models. Acta Pharmacologica Sin 35: 283-291, 2014.
18. O'neill AK, Gallegos LL, Justilien V, Garcia EL, Leitges M, Fields AP, Hall RA and Newton AC: Protein kinase C $\alpha$ promotes cell migration through a PDZ-dependent interaction with its novel substrate discs large homolog 1 (DLG1). J Biol Chem 286: 43559-43568, 2011.

19. Mason SA, Cozzi SJ, Pierce CJ, Pavey SJ, Parsons PG and Boyle GM: The induction of senescence-like growth arrest by protein kinase $\mathrm{C}$-activating diterpene esters in solid tumor cells. Invest New Drugs 28: 575-586, 2010.

20. Akakura S, Nochajski P, Gao L, Sotomayor P, Matsui S and Gelman IH: Rb-dependent cellular senescence, multinucleation and susceptibility to oncogenic transformation through PKC scaffolding by SSeCKS/AKAP12. Cell Cycle 9: 4656-4665, 2010.

21. Grealish S, Xie L, Kelly M and Dowd E: Unilateral axonal or terminal injection of 6-hydroxydopamine causes rapid-onset nigrostriatal degeneration and contralateral motor impairments in the rat. Brain Res Bull 77: 312-319, 2008.

22. Koziorowski D, Friedman A, Arosio P, Santambrogio P and Dziewulska D: ELISA reveals a difference in the structure of substantia nigra ferritin in Parkinson's disease and incidental lewy body compared to control. Parkinsonism Relat Disord 13: 214-218, 2007.

23. Itahana K, Zou Y, Itahana Y, Martinez JL, Beausejour C, Jacobs JJ, Van Lohuizen M, Band V, Campisi J and Dimri GP: Control of the replicative life span of human fibroblasts by p16 and the polycomb protein Bmi-1. Mol Cell Biol 23: 389-401, 2003.

24. West MJ, Slomianka L and Gundersen HJ: Unbiased stereological estimation of the total number of neurons in thesubdivisions of the rat hippocampus using the optical fractionator. Anat Rec 231: 482-497, 1991.

25. Pan J, Wang G, Yang HQ, Hong Z, Xiao Q, Ren RJ, Zhou HY, Bai L and Chen SD: K252a prevents nigral dopaminergic cell death induced by 6-hydroxydopamine through inhibition of both mixed-lineage kinase 3/c-Jun NH2-terminal kinase 3 (JNK3) and apoptosis-inducing kinase 1/JNK3 signaling pathways. Mol Pharmacol 72: 1607-1618, 2007.

26. German DC and Manaye KF: Midbrain dopaminergic neurons (nuclei A8, A9 and A10): Three-dimensional reconstruction in the rat. J Comp Neurol 331: 297-309, 1993.

27. Streit WJ, Braak H, Xue QS and Bechmann I: Dystrophic (senescent) rather than activated microglial cells are associated with tau pathology and likely precede neurodegeneration in Alzheimer's disease. Acta Neuropathol 118: 475-485, 2009.

28. Luo XG and Chen SD: The changing phenotype of microglia from homeostasis to disease. Transl Neurodegener 1: 9, 2012.

29. Kim YS and Joh TH: Microglia, major player in the brain inflammation: Their roles in the pathogenesis of Parkinson's disease. Exp Mol Med 38: 333-347, 2006.

30. Franceschi C, Bonafé M, Valensin S, Olivieri F, De Luca M, Ottaviani E and De Benedictis G: Inflamm-aging. An evolutionary perspective on immunosenescence. Ann N Y Acad Sci 908: 244-254, 2000.

31. Inamizu T, Chang MP and Makinodan T: Influence of age on the production and regulation of interleukin-1 in mice. Immunology 55: 447-455, 1985.

32. Corsini E, Battaini F, Lucchi L, Marinovich M, Racchi M, Govoni S and Galli CL: A defective protein kinase C anchoring system underlying age-associated impairment in TNF-alpha production in rat macrophages. J Immunol 163: 3468-3473, 1999.

33. Plackett TP, Boehmer ED, Faunce DE and Kovacs EJ: Aging and innate immune cells. J Leukoc Biol 76: 291-299, 2004.

34. Dimri GP, Lee X, Basile G, Acosta M, Scott G, Roskelley C, Medrano EE, Linskens M, Rubelj I and Pereira-Smith O: A biomarker that identifies senescent human cells in culture and in aging skin in vivo. Proc Natl Acad Sci USA 92: 9363-9367, 1995.

35. Yu HM, Zhao YM, Luo XG, Feng Y, Ren Y, Shang H, He ZY, Luo XM, Chen SD and Wang XY: Repeated lipopolysaccharide stimulation induces cellular senescence in BV2 cells. Neuroimmunomodulation 19: 131-136, 2012.

36. Petiti JP, De Paul AL, Gutiérrez S, Palmeri CM, Mukdsi JH and Torres AI: Activation of PKC epsilon induces lactotroph proliferation through ERK1/2 in response to phorbol ester. Mol Cell Endocrinol 289: 77-84, 2008

37. Inoue S, Hao Z, Elia AJ, Cescon D, Zhou L, Silvester J, Snow B, Harris IS, Sasaki M, Li WY, et al: Mule/Huwe1/Arf-BP1 suppresses Ras-driven tumorigenesis by preventing c-Myc/Miz1-mediated down-regulation of p21 and p15. Genes Dev 27: 1101-1114, 2013. 
38. Mowla S, Pinnock R, Leaner VD, Goding CR and Prince S: PMA-induced up-regulation of TBX3 is mediated by AP-1 and contributes to breast cancer cell migration. Biochem $\mathrm{J} 433$ : 145-153, 2011.

39. Serrano M, Lin AW, Mccurrach ME, Beach D and Lowe SW: Oncogenic ras provokes premature cell senescence associated with accumulation of p53 and p16INK4a. Cell 88: 593-602, 1997.

40. John R, Chand V, Chakraborty S, Jaiswal N and Nag A: DNA damage induced activation of Cygb stabilizes p53 and mediates G1 arrest. DNA Repair (Amst) 2014: 107-112, 2014.

41. Sarkisian CJ, Keister BA, Stairs DB, Boxer RB, Moody SE and Chodosh LA: Dose-dependent oncogene-induced senescence in vivo and its evasion during mammary tumorigenesis. Nat Cell Biol 9: 493-505, 2007.

42. Courtois-Cox S, Genther Williams SM, Reczek EE, Johnson BW McGillicuddy LT, Johannessen CM, Hollstein PE, MacCollin M and Cichowski K: A negative feedback signaling network underlies oncogene-induced senescence. Cancer Cell 10 459-472, 2006

43. Chinta SJ, Lieu CA, Demaria M, Laberge RM, Campisi J and Andersen JK: Environmental stress, ageing and glial cell senescence: A novel mechanistic link to Parkinson's disease? J Intern Med 273: 429-436, 2013.

44. Kumar A, Chen SH, Kadiiska MB, Hong JS, Zielonka J, Kalyanaraman B and Mason RP: Inducible nitric oxide synthase is key to peroxynitrite-mediated, LPS-induced protein radical formation in murine microglial BV2 cells. Free Radical Bio Med 73: 51-59, 2014

45. Collado M and Serrano M: Senescence in tumours: Evidence from mice and humans. Nat Rev Cancer 10: 51-57, 2010.
46. Parrinello S, Samper E, Krtolica A, Goldstein J, Melov S and Campisi J: Oxygen sensitivity severely limits the replicative lifespan of murine fibroblasts. Nat Cell Biol 5: 741-747, 2003.

47. Chen JH, Ozanne SE and Hales CN: Methods of cellular senescence induction using oxidative stress. Methods Mol Biol 371: 179-189, 2007.

48. Bitto A, Sell C, Crowe E, Lorenzini A, Malaguti M, Hrelia S and Torres C: Stress-induced senescence in human and rodent astrocytes. Exp Cell Res 316: 2961-2968, 2010.

49. Inoue Y, Matsuda T, Sugiyama KI, Izawa S and Kimura A: Genetic analysis of glutathione peroxidase in oxidative stress response of Saccharomyces cerevisiae. J Biol Chem 274: 27002-27009, 1999.

50. Kaneto H, Kajimoto Y, Fujitani Y, Matsuoka T, Sakamoto K, Matsuhisa M, Yamasaki Y and Hori M: Oxidative stress induces p21 expression in pancreatic islet cells: Possible implication in beta-cell dysfunction. Diabetologia 42: 1093-1097, 1999.

51. Croce CM: Oncogenes and cancer. N Engl J Med 358: 502-511, 2008.

52. Streit WJ and Xue QS: Human CNS immune senescence and neurodegeneration. Curr Opin Immunol 29: 93-96, 2014.

53. Lehmann AR and Carr AM: The ataxia-telangiectasia gene: A link between checkpoint controls, neurodegeneration and cancer. Trends Genet 11: 375-377, 1995.

54. Vanacore N, Spila-Alegiani S, Raschetti R and Meco G: Mortality cancer risk in parkinsonian patients: A population-based study. Neurology 52: 395-398, 1999.

55. Ong EL, Goldacre R and Goldacre M: Differential risks of cancer types in people with Parkinson's disease: A national record-linkage study. Eur J Cancer 50: 2456-2462, 2014. 